\title{
SEMI-FREDHOLM OPERATORS WITH FINITE ASCENT OR DESCENT AND PERTURBATIONS
}

\author{
VLADIMIR RAKOČEVIĆ
}

(Communicated by Palle E. T. Jorgensen)

\begin{abstract}
In this note we prove that the collection of upper (lower) semiFredholm operators with finite ascent (descent) is closed under commuting operator perturbations that belong to the perturbation class associated with the set of upper (lower) semi-Fredholm operators. Then, as a corollary we get the main result of S. Grabiner (Proc. Amer. Math. Soc. 71 (1978), 79-80).
\end{abstract}

Let $X$ be an infinite-dimensional complex Banach space and denote the set of bounded (compact) linear operators on $X$ by $B(X)(K(X))$. For $T$ in $B(X)$ throughout this paper $N(T)$ and $R(T)$ will denote, respectively, the null space and the range space of $T$. Set $N^{\infty}(T)=\bigcup_{n} N\left(T^{n}\right), R^{\infty}(T)=\bigcap_{n} R\left(T^{n}\right)$, $\alpha(T)=\operatorname{dim} N(T)$ and $\beta(T)=\operatorname{dim} X / R(T)$. Recall that an operator $T \in$ $B(X)$ is semi-Fredholm if $R(T)$ is closed and at least one of $\alpha(T)$ and $\beta(T)$ is finite. For such an operator we define an index $i(T)$ by $i(T)=\alpha(T)-$ $\beta(T)$. Let $\Phi_{+}(X) \quad\left(\Phi_{-}(X)\right)$ denote the set of upper (lower) semi-Fredholm operators, i.e., the set of semi-Fredholm operators with $\alpha(T)<\infty \quad(\beta(T)<$ $\infty)$. The perturbation classes associated with $\Phi_{+}(X)$ and $\Phi_{-}(X)$ are denoted, respectively, by $P\left(\Phi_{+}(X)\right)$ and $P\left(\Phi_{-}(X)\right)$, i.e.,

$$
P\left(\Phi_{+}(X)\right)=\left\{T \in B(X): T+S \in \Phi_{+}(X) \text { for all } S \in \Phi_{+}(X)\right\}
$$

and

$$
P\left(\Phi_{-}(X)\right)=\left\{T \in B(X): T+S \in \Phi_{-}(X) \text { for all } S \in \Phi_{-}(X)\right\} .
$$

Recall that $a(T)(d(T))$, the ascent (descent) of $T \in B(X)$, is the smallest non-negative integer $n$ such that $N\left(T^{n}\right)=N\left(T^{n+1}\right)\left(R\left(T^{n}\right)=R\left(T^{n+1}\right)\right)$. If no such $n$ exists, then $a(T)=\infty(d(T)=\infty)$. For a subset $M$ of $X$ let $\bar{M}$ denote the closure of $M$. The main result of this note is the following theorem.

Theorem 1. Suppose that $T, K \in B(X)$ and $T K=K T$. Then

$$
\begin{aligned}
& T \in \Phi_{+}(X), a(T)<\infty \text { and } K \in P\left(\Phi_{+}(X)\right) \Rightarrow a(T+K)<\infty, \\
& T \in \Phi_{-}(X), a(T)<\infty \text { and } K \in P\left(\Phi_{-}(X)\right) \Rightarrow d(T+K)<\infty .
\end{aligned}
$$

Received by the editors March 24, 1994 and, in revised form, June 16, 1994.

1991 Mathematics Subject Classification. Primary 47A53, 47A55.

Key words and phrases. Ascent, descent, semi-Fredholm. 
Proof. To prove (1.1) suppose that $T \in \Phi_{+}(X), a(T)<\infty, K \in P\left(\Phi_{+}(X)\right)$ and $T K=K T$. Set

$$
T_{\lambda}=T+\lambda K, \quad \lambda \in[0,1] .
$$

For each $\lambda \in[0,1], T_{\lambda} \in \Phi_{+}(X)$. By [2, Theorem 3], there exists $\varepsilon=\varepsilon(\lambda)>0$ such that

$$
\overline{N^{\infty}\left(T_{\lambda}\right)} \cap R^{\infty}\left(T_{\lambda}\right)=\overline{N^{\infty}\left(T_{\mu}\right)} \cap R^{\infty}\left(T_{\mu}\right)
$$

in the open disc $S(\lambda)$ with center $\lambda$ and radius $\varepsilon$. Since $[0,1]$ is compact, we can obtain a finite set $\left\{\lambda_{0}, \lambda_{1}, \ldots, \lambda_{n}\right\}$ of points on $[0,1]$ such that $\lambda_{0}=0$, $\lambda_{n}=1$ and $[0,1] \subset \bigcup_{i=0}^{n} S\left(\lambda_{i}\right)$ with $S\left(\lambda_{i}\right) \cap S\left(\lambda_{i+1}\right) \neq \emptyset$ for $i=0,1, \ldots, n-1$. Now since $a(T)<\infty$, it follows that $N^{\infty}(T) \cap R^{\infty}(T)=\overline{N^{\infty}(T)} \cap R^{\infty}(T)=\{0\}$ [6, Proposition 1.6. (i)], and by (1.3) we have that $\overline{N^{\infty}\left(T_{\mu}\right)} \cap R^{\infty}\left(T_{\mu}\right)=\{0\}$ for all $\mu$ in $S\left(\lambda_{0}\right)$. Hence, because $S\left(\lambda_{1}\right)$ overlaps $S\left(\lambda_{0}\right)$, we conclude that $\overline{N^{\infty}\left(T_{\mu}\right)} \cap R^{\infty}\left(T_{\mu}\right)=\{0\}$ for all $\mu$ in $S\left(\lambda_{1}\right)$. By proceeding along the family of disc, we finally deduce that $\overline{N^{\infty}\left(T_{\mu}\right)} \cap R^{\infty}\left(T_{\mu}\right)=\{0\}$ for all $\mu$ in $S\left(\lambda_{n}\right)$. Thus $N^{\infty}\left(T_{\lambda_{n}}\right) \cap R^{\infty}\left(T_{\lambda_{n}}\right)=\{0\}$, and again by [6, Proposition 1.6. (i)] it follows that $a(T+K)<\infty$. This completes the proof of $(1.1)$.

To prove (1.2) suppose that $T \in \Phi_{-}(X), d(T)<\infty, K \in P\left(\Phi_{-}(X)\right)$ and $T K=K T$. Then $T^{*} \in \Phi_{+}\left(X^{*}\right), a\left(T^{*}\right)<\infty, T^{*} K^{*}=K^{*} T^{*}$ and $T^{*}+\lambda K^{*} \in$ $\Phi_{+}\left(X^{*}\right), \lambda \in[0,1][1$, pp. 7-8]. Part (1.2) now follows directly from the proof of part (1.1). This completes the proof.

Let us remark that the commutativity condition in Theorem 1 is essential, even for compact $K$ [1, pp. 13-14]. In order to prove Theorem 1 we need the hypothesis that $K$ commutes with $T$ in the place where we invoke [2, Theorem 3].

Now as a corollary, we get the main result of S. Grabiner [3, Theorem 2] (see also [4, Theorem 7.9.2]). Our formulation of that result is somehow different from that of S. Grabiner's, but appropriate to Theorem 1.

Corollary 2. Suppose that $T \in B(X), K \in K(X)$ and $T K=K T$. Then

$$
\begin{aligned}
& T \in \Phi_{+}(X) \text { and } a(T)<\infty \Rightarrow a(T+K)<\infty, \\
& T \in \Phi_{-}(X) \text { and } d(T)<\infty \Rightarrow d(T+K)<\infty .
\end{aligned}
$$

Proof. By Theorem 1 and the fact that $K(X) \subset P\left(\Phi_{+}(X)\right) \cap P\left(\Phi_{-}(X)\right)$ [1, Theorem 5.6.9].

To help readers understand how far our Theorem 1 extends the result in [3], we refer them to the discussion of perturbation ideals in Sections 5.5 and 5.6, pages $95-102$ in [1]. Let us mention in particular that $P\left(\Phi_{+}(X)\right)$ includes all strictly singular operators.

Let $\sigma_{a}(T)$ and $\sigma_{d}(T)$ denote, respectively, the approximate point spectrum and the approximate defect spectrum of an element $T$ of $B(X)$. Set

$$
\sigma_{a b}(T)=\bigcap_{\substack{T K=K T \\ K \in K(X)}} \sigma_{a}(T+K) \text { and } \sigma_{d b}(T)=\bigcap_{\substack{T K=K T \\ K \in K(X)}} \sigma_{d}(T+K) .
$$

We call $\sigma_{a b}(T)$ and $\sigma_{d b}(T)$, respectively, Browder's essential approximate point spectrum of $T$ and Browder's essential approximate defect spectrum of $T$ [5], [7]. Recall that by [5, Theorem 2.1] a complex number $\lambda \notin \sigma_{a b}(T)\left(\sigma_{d b}(T)\right)$ 
if and only if $T-\lambda \in \Phi_{+}(X), i(T) \leq 0$ and $a(T-\lambda)<\infty \quad\left(T-\lambda \in \Phi_{-}(X)\right.$, $i(T) \geq 0$ and $d(T-\lambda)<\infty)$.

Finally, as a second application of Theorem 1 we have

Corollary 3. Suppose that $T \in B(X)$. Then

$$
\sigma_{a b}(T)=\bigcap_{\substack{\left.T K \bar{T}_{K \in P} K \mathcal{\Phi _ { + }}(X)\right) \\ K \in P}} \sigma_{a}(T+K)
$$

and

$$
\sigma_{d b}(T)=\bigcap_{\substack{T K K=K T \\ K \in P\left(\bar{\Phi}_{-}(X)\right)}} \sigma_{d}(T+K) .
$$

Proof. By Theorem 1 and [5, Theorem 2.1].

\section{ACKNOWLEDGMENT}

I am grateful to Professor Sandy Grabiner for helpful correspondence and conversations. The author also thanks the referee for helpful comments and suggestions concerning the paper.

\section{REFERENCES}

1. S. R. Caradus, W. E. Pfaffenberger, and B. Yood, Calkin algebras and algebras of operators on Banach spaces, Marcel Dekker, New York, 1974.

2. M. A. Goldman and S. N. Kračkovskii, Behaviour of the space of zero elements with finite-dimensional salient on the Riesz kernel under perturbations of the operator, Dokl. Akad. Nauk SSSR 221 (1975), 532-534; English transl., Soviet Math. Dokl.,16(, 1975), 370-373.

3. S. Grabiner, Ascent, descent, and compact perturbations, Proc. Amer. Math. Soc. 71 (1978), 79-80.

4. R. Harte, Invertibility and singularity for bounded linear operators, Marcel Dekker, New York and Basel, 1988.

5. V. Rakočević, Approximate point spectrum and commuting compact perturbations, Glasgow Math. J. 28 (1986), 193-198.

6. T. T. West, A Riesz-Schauder theorem for semi-Fredholm operators, Proc. Roy. Irish Acad. Sect. A 87 (1987), 137-146.

7. J. Zamánek, Compressions and the Weyl-Browder spectra, Proc. Roy. Irish Acad. Sect. A 86 (1986), 57-62.

Department of Mathematics, University of Niš, Faculty of Philosophy, Ćirila and Metodija 2, 18000 Niš, Yugoslavia 